\title{
Diversity and composition of soil bacterial community respond to $A$. palmeri invasion under heterogeneous habitats
}

\section{Mei Zhang ( $\square$ zhangmeinku@163.com )}

Nankai University College of Life Sciences

Jianhua Han

Tianjin Agricultural Ecological Environment Monitoring and Quality of Agricultural Products Testing Centre

\section{Xueying Li}

Nankai University college of life sciences

\section{Pufan Zheng}

Nankai University

\section{Zhenlu Qiu}

Nankai University College of Life Sciences

\section{Lili Tang}

Nankai University College of Life Sciences

\section{Tongtong Wang}

Nankai University College of Life Sciences

\section{Fuchen Shi}

Nankai University College of Life Sciences https://orcid.org/0000-0002-0385-5923

\section{Research Article}

Keywords: Plant invasion, Amaranthus palmeri, Soil bacterial community, Heterogeneous habitat, Fulllength 16S rRNA sequencing

Posted Date: June 22nd, 2021

DOl: https://doi.org/10.21203/rs.3.rs-595834/v1

License: (c) (1) This work is licensed under a Creative Commons Attribution 4.0 International License. Read Full License 


\section{Abstract}

The impact of $A$. palmeri invasion on soil bacterial community under different habitats is unclear. In this work, the influence of $A$. palmeri invasion on soil bacterial diversity and community structure were investigated using full-length 16S rRNA sequencing technology under four typical habitats of riverbank $(A)$, roadside (B), wasteland (C) and farmland (D). A two-way ANOVA analysis showed that habitat, invasion and the interaction of them had little effect on alpha diversity, expect for habitat factor had a significant effect on Simpson indices $(P<0.05)$. NMDS analysis demonstrated that soil bacterial community structures among different invasive habitats were clearly distinguished. In addition, the most abundant phyla in the non-invasive plots were Proteobacteria, Planctomycetes and Gemmatimonadetes. However, the third predominant phyla converted from Bacteroidetes to Gemmatimonadetes with the invasion of $A$. palmeri. LEfSe analysis revealed that the core microbiome, Burkholderiaceae and Betaproteobacteriales (riverbank habitat), Gemmatimonadetes and Gemmatimonadaceae (wasteland habitat), Sphingomonas_sediminicola (roadside habitat), Nitrosomonadaceae (farmland habitat), which played important roles in facilitating the establishment of $A$. palmeri to heterogeneous habitats.

\section{Introduction}

Amaranthus palmeri (A. palmeri), native to America, belongs to the Amaranthaceae, amaranth plant (Ehleringer 1983). Some characteristics make it a global malignant and troublesome weed, for example, $\mathrm{C}_{4}$ photosynthetic pathway, whose photosynthetic rate is 3-4 times higher than soybean and cotton (Gibson 1998; Palma-Bautista et al. 2019). Extremely high reproductive capacity and growth rate, each plant can produce 600,000 seeds and the plant height can reach 2-3m under ideal conditions (Keeley et al. 1987; Ward et al. 2013). Dioecism, high stress tolerance and genetic variation make it harder to control (Bond and Oliver 2006; Oliveira et al. 2018). Moreover, A. palmeri has developed resistance to a variety of herbicide modes in many states of America, such as action-dinitroanilines, ALS-inhibitors, photosystem II inhibitors and glycines-with GR (Ahmed 2011; Heap 2014).

A. palmeri was discovered in Fengtai District, Beijing in 1985, which was the first time that $A$. palmeri appeared in China. After nearly 30 years of latent transmission, it has shown an explosive invasion and diffusion trend in North China in recent years. It rapidly invades into farmland, orchards and other economic crops growing areas, competing with cash crops for nutrients, water, and light, which causes a huge threat and damage to the local agricultural ecosystem and biodiversity security. There are few studies on A. palmeri in China, mainly including: Li et al. (2015) predicted the potential distribution of $A$. palmeri in China. Cao et al. (2015) showed that phenotypic variation (such as earlier flowering time of high-latitude populations) could promote the environmental adaptive evolution of $A$. palmeri and expand its suitable distribution in China. The previous study on phenotypic plasticity was carried out in our research group (Zhang et al. 2020).

The invasion mechanism of alien plants is very complex, including increased competitive ability, allelopathy, physical, chemical and microbial changes in rhizosphere, etc (Zhang et al. 2009; Fang et al. 
2019; Zhang et al. 2018). In recent years, it has become a new research idea and an important development trend to study the invasion mechanism of alien plants from the view of plant-soil-microbial community. The invasion of Ageratina adenophora will change the diversity and community structure of soil microorganisms, causing the accumulation of beneficial functional bacteria and the change of soil enzyme activities, forming a micro-ecological environment conducive to its invasion and expansion (Niu et al. 2007; Xu et al. 2012; Hu et al. 2019). Smooth brome increases the richness and evenness of soil bacteria, and the most important role is to selectively inhibit the dominant bacteria and increase the relative abundance of rare bacteria (Piper et al. 2015). The invasion of Robinia pseudoacacia alters soil bacterial abundance, which is mainly driven by Actinobacteria, Gemmatimonadetes and Nitrospirae (Liu et al. 2018). Mikania Micrantha can change the interaction between microbial and microfaunal, thereby stimulating potassium release (potassium-solubilizing bacteria) compared to native plants (Sun et al. 2019). Cirsium Arvense degrades the performance of some native plants by altering soil microbes, but its own performance does not change, which makes competitive advantages of Cirsium (Verbeek and Kotanen 2019). Cheng et al. (2019) found many rhizosphere and endophytic bacteria related to phosphate solubilization (Brevundimonas diminuta), nitrogen fixation (Rhizobium leguminosarum) and extreme environment tolerance (Exiguobacterium sibiricum) of invasive plant Senecio vulgaris, those bacteria may facilitate the invasive ability of $S$. vulgaris. However, the influence of soil microbes on the invasion of $A$. palmeri is still unclear. Therefore, it is of great theoretical and practical significance to reveal the invasion mechanism of $A$. palmeri from the perspective of soil microorganisms, thereby inhibiting its further diffusion and spread.

In this research, field experiment combined with full-length 16S rRNA sequencing technology were used to study soil microbial diversity and composition under four major invasion habitats. We hypothesized that soil bacterial communities would display distinct differences across heterogeneous invasive habitats. Furthermore, there would be different types of potential core microbiome that may contribute to the successful establishment of $A$. palmeri to new locations.

\section{Materials And Methods}

\section{Study site and sampling collection}

According to filed investigations, A. palmeri has four main invaded habitats, including riverbank (A), roadside (B), wasteland (C) and farmland (D) (Fig. 1). In August, 2019, we collected soil samples in the four major habitats. The sampling site is located in Wuqing District of Tianjin, North of China $\left(117^{\circ} 16 \mathbb{E} \bigotimes\right.$ $39^{\circ} 59 \llbracket \mathrm{N}$ ), which belongs to temperate semi-humid continental monsoon climate, with an average annual temperature of $11.6^{\circ} \mathrm{C}$, and an annual precipitation of $606 \mathrm{~mm}$. This area is an ideal sampling site to study the effect of $A$. palmeri invasion on soil bacterial community, because it has suffered from severe invasion and formed single-dominant community in various habitats. The main accompanying herbs are Setaria Viridis (L.) Beauv., Chenopodium album L., Chloris virgata Sw., Zea Mays L. etc. 
Four habitat sites including riverbank (A), roadside (B), wasteland (C) and farmland (D) were selected (Fig. 1). Three invaded plots (coverage more than $70 \%$ ) and three non-invaded plots (control plot) were randomly designed from each of the four habitats, the six plots were 3-5m away from each other. Each plot was set up with $2 \times 2 \mathrm{~m}$ quadrats. At each quadrat, five soil cores were collected by an auger (diameter of $2.5 \mathrm{~cm}$ ) after removing the litter layer to collect the soil adjacent to the stem of A. palmeri and its host, five cores were mixed into one sample in each quadrat.

A total of 24 soil samples with 4 (habitat) $\times 2$ (soil type) $\times 3$ (repeat) were collected. Soil samples were placed on ice for transport to the laboratory. Samples were and homogenized and sieved to remove visible roots and stones, then immediately stored at $-20^{\circ} \mathrm{C}$ prior to DNA extraction.

\section{Bacterial diversity assay}

The diversity of the bacterial communities was analyzed using single molecule real-time (SMRT) PacBio sequencing technology (Pacific Biosciences, Menlo Park, CA, USA). Genomic DNA was extracted using PowerSoil ${ }^{\circledR}$ DNA Isolation kit (Mo Bio, Solanan Beach, CA, USA) following the instructions provided by the manufacturer. Primers 27F (5'-AGAGTTTGATCCTGGCTCAG-3') and 1492R (5'-

GGTTACCTTGTTACGACTT-3') were designed to amplify the full length of 16S rRNA gene of the bacteria. Each 50uL polymerase chain reaction (PCR) amplification system includes: 40-60ng DNA, 2.5 $\mu \mathrm{l}$ * $\mathrm{Vn}$ $\mathrm{F}(10 \mu \mathrm{M}), 2.5 \mu \mathrm{l} * \mathrm{Vn} \mathrm{R}(10 \mu \mathrm{M}), 1 \mu \mathrm{l} \mathrm{KOD} \mathrm{FX}$ Neo(TOYOBO), $25 \mu \mathrm{l} \mathrm{KOD} \mathrm{FX} \mathrm{Neo} \mathrm{Buf(2X)} \mathrm{and} 10 \mu \mathrm{l} 2 \mathrm{Mm}$ dNTP; $\mathrm{ddH}_{2} \mathrm{O}$ was used to supplement the total system to $50 \mu \mathrm{L}$. Cycling conditions consisted of initial denaturation at $95^{\circ} \mathrm{C}$ for $5 \mathrm{~min} ; 30$ cycles including $95^{\circ} \mathrm{C}$ for $30 \mathrm{sec}, 50^{\circ} \mathrm{C}$ for $30 \mathrm{sec}$, and $72^{\circ} \mathrm{C}$ for $1 \mathrm{~min} / 1 \mathrm{~kb}$; elongation at $72^{\circ} \mathrm{C}$ for $5 \mathrm{~min}$. PCR amplification products were separated by agarose gel electrophoresis and purified by MagicPure Size Selection DNA Beads (TransGen Biotech, Beijing, CN). Sequencing libraries were built by the ligation of barcoded PCR products and SMRT bell adapters, then the libraries were sequenced by PacBio Sequel.

Circular consensus sequencing (CCS) were obtained by correcting the original offline subreads using SMRT Analysis Software (Pacific Biosciences, USA). Then, CCS sequences of different samples were identified by barcode sequence and chimera were removed to obtain high-quality CCS sequences according to Lima v1.7.0 software. The remaining sequences were clustered at $97 \%$ similarity and assigned into operational taxonomic units (OTUs) at 0.005\% threshold (Quast et al. 2013; Kõljalg et al. 2013)

\section{Statistical analysis}

Alpha diversity indices (ACE, Chao1, Shannon and Simpson) were calculated using Mothur version v.1.30 software. Two-way analysis of variance (ANOVA) using SPSS 20.0 was to evaluate the effect of invasion, habitat and the interaction between them on alpha diversity indices of bacterial community. To visualize the clustering of different habitats, non-metric multi-dimensional scaling (NMDS) was constructed based on binary-jaccard dissimilarities. Furthermore, the linear discriminant analysis (LDA) effect size (LEfSe) method was used to discover the potential biomarkers for different statistical groups of treatments. A 
significance Kruskal-Wallis test of 0.05 and an LDA score threshold of 4 were used for all biomarkers evaluated in this study. All figures were generated in the R statistical environment.

\section{Results}

\section{Sequencing results}

Sequencing and identifying of 24 samples were performed by barcode, and a total of 180441 CCS sequences were obtained. Each sample produced at least 3356 CCS sequences, with an average of 7518 CCS sequences.

Venn diagram can directly reflect the difference and overlap of bacterial OUTs. The four habitats of riverbank (A), roadside (B), wasteland (C) and farmland (D) detected 1814, 1647, 1593, and 1608 bacterial OUTs in the invaded plots, and 2105, 1615, 1909, 1448 bacterial OUTs in the non-invaded plots, respectively. There were 1285 and 1021 identical bacterial OUTs in invaded and non-invaded plots of the four habitats, In the invaded plot, 314(A), 145(B), 117(C), and 240(D) OUTs were solely detected, additionally, 213(A), 201(B), 127(C), and 104(D) bacterial OUTs were exclusively observed in the noninvaded areas, respectively (Fig. 2).

\section{Diversity of bacterial communities under four habitats of $A$. palmeri}

Chao1, ACE, Shannon and Simpson metrics were used to calculate bacterial alpha diversity. Simpson indices were higher in $A$ habitat and lower in $C$ and $D$ habitats. In contrast, $C$ habitat had the highest Shannon indices (both invaded and non-invaded plots), Chao1 and ACE richness (non-invaded plot). Based on Two-Way ANOVA, in addition to habitat factors had a significant effect on Simpson indices, there was no significant difference in alpha diversity between invasive and non-invasive plots of different habitats (Table 1).

Table 1. Alpha diversity of bacterial community in the invasive and non-invasive plots under four habitats. 


\begin{tabular}{ccccc}
\hline AN & $0.0036 \pm 0.002 \mathrm{ab}$ & $6.458 \pm 0.300 \mathrm{ab}$ & $1729.2 \pm 281 \mathrm{ab}$ & $1693.3 \pm 308 \mathrm{ab}$ \\
AY & $0.0045 \pm 0.001 \mathrm{a}$ & $6.145 \pm 0.111 \mathrm{~b}$ & $1455.9 \pm 56 \mathrm{bc}$ & $1424.7 \pm 63 \mathrm{ab}$ \\
BN & $0.0021 \pm 0.001 \mathrm{bc}$ & $6.484 \pm 0.278 \mathrm{ab}$ & $1691.2 \pm 307 \mathrm{abc}$ & $1596.5 \pm 268 \mathrm{ab}$ \\
BY & $0.0032 \pm 0.001 \mathrm{abc}$ & $6.270 \pm 0.173 \mathrm{ab}$ & $1343.2 \pm 179 \mathrm{c}$ & $1312.2 \pm 188 \mathrm{~b}$ \\
$\mathrm{CN}$ & $0.0022 \pm 0.000 \mathrm{bc}$ & $6.601 \pm 0.126 \mathrm{a}$ & $1894.6 \pm 195 \mathrm{a}$ & $1790.8 \pm 260 \mathrm{a}$ \\
CY & $0.0017 \pm 0.000 \mathrm{c}$ & $6.582 \pm 0.187 \mathrm{a}$ & $1675.9 \pm 172 \mathrm{abc}$ & $1577.1 \pm 177 \mathrm{ab}$ \\
DN & $0.0021 \pm 0.000 \mathrm{bc}$ & $6.411 \pm 0.042 \mathrm{ab}$ & $1497.9 \pm 14 \mathrm{bc}$ & $1451.9 \pm 87 \mathrm{ab}$ \\
DY & $0.0016 \pm 0.000 \mathrm{c}$ & $6.432 \pm 0.351 \mathrm{ab}$ & $1535.4 \pm 157 \mathrm{abc}$ & $1479.7 \pm 207 \mathrm{ab}$ \\
Habitat & $* *$ & $\mathrm{~ns}$ & $\mathrm{~ns}$ & $\mathrm{~ns}$ \\
Invasion & $\mathrm{ns}$ & $\mathrm{ns}$ & $\mathrm{ns}$ & $\mathrm{ns}$ \\
HabitatxInvasion & $\mathrm{ns}$ & $\mathrm{ns}$ & $\mathrm{ns}$ & $\mathrm{ns}$
\end{tabular}

A, B, C, D indicate riverbank, roadside, wasteland and farmland habitats, respectively. $\mathrm{N}$ indicates non-invaded plot, $\mathrm{Y}$ indicates invaded plot. Data are mean values \pm SEM, Different letters denote significant differences $(P<0.05)$.

NMDS analysis at the OTU level (binary-jaccard dissimilarities) were used to compare soil bacterial community structures of four different habitats (Fig. 3). Bacterial communities can be divided into four clusters based on the four habitats, which showed a visible difference amongst the four habitats of microbial community compositions. Moreover, the bacterial communities in $\mathrm{D}$ habitat were distant from those in $A$ and $B$ habitats, and the distance between $C$ and $D$ habitat was closer.

\section{Phylum-level taxonomic composition of bacterial community of the four habitats}

The bacterial phyla composition showed that Proteobacteria, Planctomycetes, Bacteroidetes, Gemmatimonadetes and Acidobacteria were the most dominant phylum in all habitats (Fig. 4). Proteobacteria were the most abundant phyla, with a relative abundance of $37.91 \%, 29.71 \%, 27.15 \%$, $27.42 \%$ in the non-invaded plots, and a relative abundance of $42.75 \%, 26.08 \%, 30.35 \%, 28.41 \%$ in the invaded plots of the $A, B, C$ and $D$ habitats, respectively. Planctomycetes were the second dominant phylum, with a relative abundance of $7.61 \%, 13.41 \%, 19.24 \%, 20.57 \%$ in the non-invaded plots, and a relative abundance of $5.38 \%, 21.79 \%, 19.78 \%, 21.34 \%$ in the invaded plots of the $A, B, C$ and $D$ habitats, respectively. Invasion of $A$. palmeri increased the abundance of Proteobacteria in $A$ and $C$ habitats by $12.77 \%$ and $11.79 \%$, enriched the abundance of Planctomycetes in B habitats by $62.49 \%$, decreased the abundance of Planctomycetes by $41.45 \%$ in A habitat. More importantly, the third bacterial phyla were changed from Gemmatimonadetes to Bacteroidetes due to the invasion of $A$. palmeri. These changes were mainly associated with the enrichment of Bacteroidetes in A, C and D habitats by $134.47 \%, 17.25 \%$ and $42.04 \%$, and the decrease of Gemmatimonadetes by in $61.18 \%, 27.71$ and $9.35 \%$ in A, B and C habitats. The fourth dominant phylum were Acidobacteria with a significant decrease of $48.89 \%$ under the invasion of $A$. palmeri of $\mathrm{A}$ habitat. 


\section{Determination of biomarker and core and core microorganisms in the four habitats of $A$. palmeri}

The LEfSe (Linear Discriminate Analysis Effect Size) analysis were used to identify the most significant biomarker bacterial microorganisms in the four habitats (LDA>4.0). The cladogram from the inside to the outside represented the taxonomic level from phylum to species. In the invaded plot, the LDA results reveled 15, 1, 7 and 8 phylotypes of bacteria in the riverbank, roadside, wasteland and farmland habitats, respectively. For non-invaded plot, 7, 10, 2 and 6 phylotypes of bacteria were detected in the riverbank, roadside, wasteland and farmland habitats, respectively (Fig. 5).

Compared to non-invasive plots, the relative abundance of Betaproteobacteriales (order), Burkholderiaceae (family), Saccharimonadaceae (family), Xanthomonadaceae (family), Flavisolibacter (genus); Candidatus_Saccharimonas (genus) and Flavisolibacter_sp (species) were significantly increased with the invasion of $A$. palmeri under riverbank habitat. In roadside habitat, Sphingomonas_sediminicola (species) was the only representative taxa detected in A. palmeri invasive plot compared to non-invasive plot. In wasteland habitat, Gemmatimonadetes (class) and Gemmatimonadaceae (family) were significantly more abundant under the invasion of $A$. palmeri. Meanwhile, Nitrosomonadaceae and Phycisphaeraceae Family had a higher relative abundance in the invasive plot of farmland habitat.

\section{Discussion}

Results of numerous studies have confirmed that soil microbial communities play a very important role in the process of alien plants invasion (Piper et al. 2015; Aleksandra et al. 2019; Zhao et al. 2019). Studying the impact of $A$. palmeri on soil microbial diversity under different habitats is an important way to reveal its environmental adaptation strategy of invasion. In this study, full-length 16S rRNA sequencing was used to analyze the impact of $A$. palmeri invasion on the structure and diversity of soil bacterial communities.

This study found that invasion, habitat and the interaction of them had no significant effect on alpha diversity indices (Chao1, ACE, Shannon, Simpson) of soil bacteria (Table 1). This result was inconsistent with most studies, but similar to Kamutando's research on invasive tree Acacia dealbata. Kamutando believed that microbial community analysis methods were not well suited to accurately estimate microbial richness, and diversity indices need to be applied judiciously in studies on microbial community ecology and biodiversity (Bent and Forney 2008; Kamutando et al. 2017). Therefore, the combination of multiple methods may lead to an optimal balance between resources required and information gained (Bent and Forney 2008; Zhou et al. 2007).

Proteobacteria, Planctomycetes, Bacteroidetes, Gemmatimonadetes and Acidobacteria were the most dominant phylum in all habitats (Fig. 4). These bacteria have also been widely reported in the invasion areas of other alien plants, including Japanese barberry, Acacia dealbata and Cirsium arvense (Kamutando et al. 2017; Coats et al. 2014; Nunes et al. 2019). Proteobacteria were the most dominant 
bacterial groups in this study, confirmed that Proteobacteria are the dominant bacterial community in terrestrial soil ecosystem (Bazylinski et al. 2013). Compared to non-invasive plot, Proteobacteria that have fast growth rates increased the relative abundance in riverbank habitat. While, Acidobacteria and Planctomycetes that have slower growth rates decreased the relative abundance in this habitat respond to the invasion of $A$. palmeri (Zeng 2016; Beckers et al. 2017; Ren 2020). This result was consistent with the copiotrophic hypothesis (Castro et al. 2010; Beckers et al. 2017). However, abundance of Planctomycetes enriched by a high proportion of $62.49 \%$ in roadside habitat, which may be explained by previous reports that Planctomycetes play important roles in nitrogen and carbon cycling (Nie et al. 2015; Woebken et al. 2007). This result showed that the microbial groups under the same phylum exhibited different functions, therefore, it is particularly important to analyze the core microbiome in different invasive habitats of $A$. palmeri in combination with specific genus or species. Moreover, the third bacterial phyla were changed from Gemmatimonadetes to Bacteroidetes due to the invasion of $A$. palmeri. These changes were mainly associated with the enrichment of Bacteroidetes by $134.47 \%, 17.25 \%$ and $42.04 \%$ in $A, C$ and $D$ habitats. Bacteroides are a kind of microorganisms that participate in nitrogen metabolism and labile carbon degradation. Therefore, the increase of Bacteroides may lead to the increase of soil organic carbon (Wu et al., 2017; Wang 2020).

LEfSe analysis revealed that the invasive soil bacterial communities were mainly mediated by Burkholderiaceae and Betaproteobacteriales of riverbank habitat, Gemmatimonadetes and Gemmatimonadaceae of wasteland habitat, Sphingomonas_sediminicola of roadside habitat, Nitrosomonadaceae of farmland habitat. Those of Betaproteobacteriales, Burkholderiaceae and Nitrosomonadaceae belong to Proteobacteria and all of them have been reported to have positive ecological roles in environmental ecosystems (Berendsen et al. 2012; Aguirre-von-Wobeser 2018; Lv 2014). For instance, the ability to fix nitrogen, ammonia-oxidizing, protect plant against soil pathogens (Benítez 2009; Pedrosa 2011; Berendsen et al. 2012; Zwetsloot 2020). Sphingomonas_sediminicola was first isolated from freshwater sediment in 2013 (An, 2013), Sphingomonas spp. have shown a positive effect on plant growth promotion (Fuentes, 2020). It was worth noting that, although the abundance of Gemmatimonadetes showed a downward trend in the invasive plots compared with non-invasive plots of A. palmeri under wasteland habitat, Gemmatimonadetes and Gemmatimonadaceae were still the dominant taxon in this habitat, indicating that those microorganisms played an important role in the invasion of $A$. palmeri into wasteland. In summary, our study showed that the invasion of $A$. palmeri significantly changed soil microbial community structure, and the core microbiome exhibited distinct differences responding to heterogeneous habitats.

\section{Declarations}

\section{ACKNOWLEDGMENTS}

This research was financially supported by the project of National Forestry and Grassland Administration, China (No. KJZXSA2018021), as well as the project of Tianjin Agricultural Committee, China (No.

ITTFPRS2018001). 


\section{CONFILCT OF INTREST}

The authors declare no conflict of interest.

\section{AUTHOR CONTRIBUTIONS}

Mei Zhang: Conceptualization (Lead); Data curation (Lead); Formal analysis (Lead); Investigation (Equal); Methodology (Equal); Writing-original draft (Lead). Jian-hua Han: Project administration (Lead); Supervision (Supporting). Xue-ying Li: Investigation (Equal); Writing-review and editing (Equal). Pu-fan Zheng: Investigation (Equal). Zhen-lu Qiu: Writing-review and editing (Equal). Li-li Tang: Writing-review and editing (Equal); Tong-tong Wang: Investigation (Equal); Fu-chen Shi: Funding acquisition (Lead); Methodology (Lead); Project administration (Lead); Supervision (Lead).

\section{ETHICS STATEMENT}

None required.

\section{DATA AVAILABILITY STATEMENT}

All data generated or analyzed during this study are included in this published article.

\section{References}

An DS, Liu QM, Lee HG, Jung MS, Kim SC, Lee ST (2013) Sphingomonas ginsengisoli sp. nov. and sphingomonas sediminicola sp. nov. Int J Syst Evol Micr 63(Pt 2): 496-501

Aguirre-Von-Wobeser E, Rocha-Estrada J, Shapiro LR, Mayra DLT (2018) Enrichment of Verrucomicrobia, Actinobacteria and Burkholderiales drives selection of bacterial community from soil by maize roots in a traditional milpa agroecosystem. PLoS ONE 13(12): e0208852

Ahmed AMAT (2011) Control, assessment and glyphosate resistance of Palmer Amaranth (Amaranthus palmeri S. Wats) in Virginia. MSc Thesis, Virginia Polytechnic Institute and State University

Aleksandra W, Crone EE, Zwolak R (2019) Differential impacts of soil microbes on native and co-occurring invasive tree species. Ecosphere 10: e02802

Bazylinski DA, Williams TJ, Lefevre CT, Berg RJ, Zhang CL, Bowser SS, Dean AJ, Beveridge TJ (2013) Magnetococcus marinus gen. nov., sp. nov., a marine, magnetotactic bacterium that represents a novel lineage (Magnetococcaceae fam. nov., Magnetococcales ord. nov.) at the base of the Alphaproteobacteria. Int J Syst Evol Micr 63: 801-808

Beckers B, Beeck MOD, Weyens N, Boerjan W, Vangronsveld J (2017) Structural variability and niche differentiation in the rhizosphere and endosphere bacterial microbiome of field-grown poplar trees. Microbiome 5: 25 
Bent SJ, Forney LJ (2008) The tragedy of the uncommon: understanding limitations in the analysis of microbial diversity. Isme J 2: 689-695

Berendsen RL, Pieterse CMJ, Bakker PAHM (2012) The rhizosphere microbiome and plant health. Trends Plant Sci 17: 478-486

Bond JA, Oliver LR (2006) Comparative growth of Palmer amaranth (Amaranthus palmeri) accessions. Weed Sci 54: 121-126

Cao JJ, Wang R, Li YG, Zhang GF, Guo JY, Wan FH. (20150 The phenotypic variation and environmental adaptability among different geographical populations of Amaranthus palmeri in China. Plant Quarantine 34: 25-31 (In Chinese)

Castro HF, Classen AT, Austin EE, Norby RJ, Schadt CW (2010) Soil microbial community responses to multiple experimental climate change drivers. Appl Environ Microb 76: 999-1007

Cheng DD, Tian ZS, Feng L, Xu L, Wang HM (2019) Diversity analysis of the rhizospheric and endophytic bacterial communities of Senecio vulgaris L. (Asteraceae) in an invasive range. PeerJ 6, e6162

Coats VC, Pelletreau KN, Rumpho, M.E (2014) Amplicon pyrosequencing reveals the soil microbial diversity associated with invasive Japanese barberry (Berberis thunbergii DC.). Mol Ecol 23: 1318-1332

Ehleringer J (1983) Ecophysiology of Amaranthus palmeri, a sonoran desert summer annual. Oeologica $57(1-2), 107-112$

Fang K, Chen L, Zhou J, Yang ZP, Dong XF, Zhang, HB. (2019) Plant-soil-foliage feedbacks on seed germination and seedling growth of the invasive plant Ageratina Adenophora. P Roy Soc B-Biol Sci 286(1917): 20191520

Fuentes A, Herrera H, Charles TC, Arriagada C. (2020) Fungal and bacterial microbiome associated with the rhizosphere of native plants from the Atacama desert. Microorganisms 8(2): 209

Gibson A C (1998) Photosynthetic Organs of Desert Plants: Structural designs of nonsucculent desert plants cast doubt on the popular view that saving water is the key strategy. Bioscience $48(11)$

Heap I. (2014) Herbicide resistant weeds. Intergr Pest Manag Rev 3: 281-301

Hu CH, Lei YB, Tan YH, Sun XC, Xu H, Liu CQ, Liu XY (2019) Plant nitrogen and phosphorus utilization under invasive pressure in a montane ecosystem of tropical China. $\mathrm{J}$ Ecol 107: 372-386

Kamutando CN, Vikram S, Kamgan-Nkuekam G, Makhalanyane TP, Greve M, Valverde, A (2017) Soil nutritional status and biogeography influence rhizosphere microbial communities associated with the invasive tree Acacia dealbata. Sci Rep-UK 7: 1-8 
Keeley PE, Carter CH, Thullen RJ (1987) Influence of planting date on growth of Palmer Amaranth (Amaranthus palmeri). Weed Sci 35(2): 199-204

Kõljalg U, Nilsson RH, Abarenkov K, Tedersoo L, Taylor AF, Bahram M, Bates ST, Bruns TD, BengtssonPalme J, Callaghan TM (2013) Towards a unified paradigm for sequence-based identification of fungi. Mol Ecol 22: 5271-5277

Li HQ, Zhao L, Zhu PW, Zhang Y, Zhu GP, Liu Q (2015) Potential distribution of invasive palmer amaranth in China (Amaranthus palmeri). Journal of Tianjin Normal University 35: 57-61. (In Chinese)

Liu JL, Yang ZL, Dang P, Zhu HL, Gao Y, Ha VN, Zhao Z (2018) Response of soil microbial community dynamics to Robinia pseudoacacia L. afforestation in the loess plateau: a chronosequence approach. Plant Soil 423(1): 327-338

Lv XF, Yu JB, Fu YQ, Ma B, Qu FZ, Ning K, Wu H F (2014) A Meta-Analysis of the bacterial and archaeal diversity observed in wetland soils. Sci World J 437684: 1-13

Nie SA, Li H, Yang XR, Zhang ZJ, Weng BS, Huang FY, Zhu GB, Zhu YG( 2015) Nitrogen loss by anaerobic oxidation of ammonium in rice rhizosphere. ISME J 25: 1-9

Nunes KA, Fitzpatrick CR, Kotanen PM (2019) Soil biota composition and the performance of a noxious weed across its invaded range. Ecography 42(10): 1-11

Oliveira MC, Gaines TA, Patterson EL, Jhala AJ, Irmak S, Amundsen K, Stevan ZK (2018) Interspecific and intraspecific transference of metabolism-based mesotrione resistance in dioecious weedy Amaranthus. Plant J 96(5): 1051-1063

Palma-Bautista C, Torra J, García MJ, Bracamonte E, Rojano-Delgado AM, Alcántara-de la Cruz R, Pradoet RD (2019) Reduced absorption and impaired translocation endows glyphosate resistance in Amaranthus palmeri harvested in glyphosate-resistant soybean from Argentina. J Agr Food Chem 67: 1052-1060

Pedrosa FO, Monteiro RA, Wassem R, Cruz LM, Ayub RA, Colauto NB, Fernandez MA, Fungaro MHP, Grisard EC, Hungria M (2011) Genome of Herbaspirillum seropedicae strain SmR1, a specialized diazotrophic endophyte of tropical grasses. PLoS Genet 7(5): e1002064

Piper CL, Siciliano SD, Winsley T, Lamb EG (2015) Smooth brome invasion increases rare soil bacterial species prevalence, bacterial species richness and evenness. J Ecol 103(2): 386-396

Quast C, Pruesse E, Yilmaz P, Gerken J, Schweer T, Yarza P, Peplies J, Glöckner FO (2013) The SILVA ribosomal RNA gene database project: improved data processing and web-based tools. Nucleic acids res 41: $590-596$

Ren AT, Abbott LK, Chen Y, Xiong YC, Mickan BS (2020) Nutrient recovery from anaerobic digestion of food waste: impacts of digestate on plant growth and rhizosphere bacterial community composition and 
potential function in ryegrass. Biol and Fert Soils

Sun F, Ou Q J, Yu H X, Li N, Peng CL (2019) The invasive plant Mikania micrantha affects the soil foodweb and plant-soil nutrient contents in orchards. Soil Biol Biochem139: 107630

Verbeek JD, Kotanen PM (2019) Soil-mediated impacts of an invasive thistle inhibit the recruitment of certain native plants. Oecologia 190: 619-628

Wang XJ, Zhang ZC, Yu ZQ, Shen GF, Tao S (2020) Composition and diversity of soil microbial communities in the alpine wetland and alpine forest ecosystems on the Tibetan Plateau. Sci Total Environ 747, 141358

Ward SM, Webster TM, Steckel LE (2013) Palmer Amaranth (Amaranthus palmeri): A Review. Weed Technol 27(1): 12-27

Woebken D, Teeling H, Wecker P, Dumitriu A, Kostadinov I, Delong EF, Glöckner FO (2007) Fosmids of novel marine planctomycetes from the Namibian and Oregon coast upwelling systems and their crosscomparison with Planctomycete genomes. ISME J 1(5), 419-435

Wu XD, Xu HY, Liu GM, Ma XL, Mu CC, Zhao L (2017) Bacterial communities in the upper soil layers in the permafrost regions on the Qinghai-Tibetan plateau. Appl Soil Ecol 120(8), 81-88

Xu CW, Yang MZ, Chen YJ, Chen LM, Zhang DZ (2012) Changes in non-symbiotic nitrogen-fixing bacteria inhabiting rhizosphere soils of an invasive plant Ageratina Adenophora. Appl Soil Ecol, 54(none): 32-38

Zeng J, Liu X, Song L, Lin XG, Zhang HY, Shen CC (2016) Nitrogen fertilization directly affects soil bacterial diversity and indirectly affects bacterial community composition. Soil Biol Biochem 92: 41-49

Zhang CB, Wang J, Qian BY, Li WH (2009) Effects of the invader Solidago canadensis on soil properties. Appl Soil Ecol 43(2-3): 163-169

Zhang G, Bai J, Jia J, Wang W, Zhao QQ, Lu QQ (2018) Shifts of soil microbial community composition along a short-term invasion chronosequence of Spartina alterniflora in a Chinese estuary. Sci Total Environ 657: 222-233

Zhang M, Ma K X, Liu T, Tang LL, Khan AA, Yang T, Shi, FC (2019) Responses in phenotypic plasticity of Amaranthus palmeri and Polygonum orientale to soil factors under different habitats. CLEAN - Soil, Air, Water 48(1): 1-8

Zhao MX, Lu XF, Zhao HX, Yang YF, Wan FH (2019) Ageratina adenophora invasions are associated with microbially mediated differences in biogeochemical cycles. Sci Total Environ 677: 47-56

Zhou X, Brown CJ, Abdo Z, Davis CC, Hansmann MA, Joyce P, Foster JA, Forney LJ (2007) Differences in the composition of vaginal microbial communities found in healthy Caucasian and black women. ISME $J$ 
Zwetsloot MJ, Juana Muñoz Ucros, Wickings K, Wilhelm RC, Bauerle TL (2020) Prevalent root-derived phenolics drive shifts in microbial community composition and prime decomposition in forest soil. Soil Biol and Biochem 145

\section{Figures}
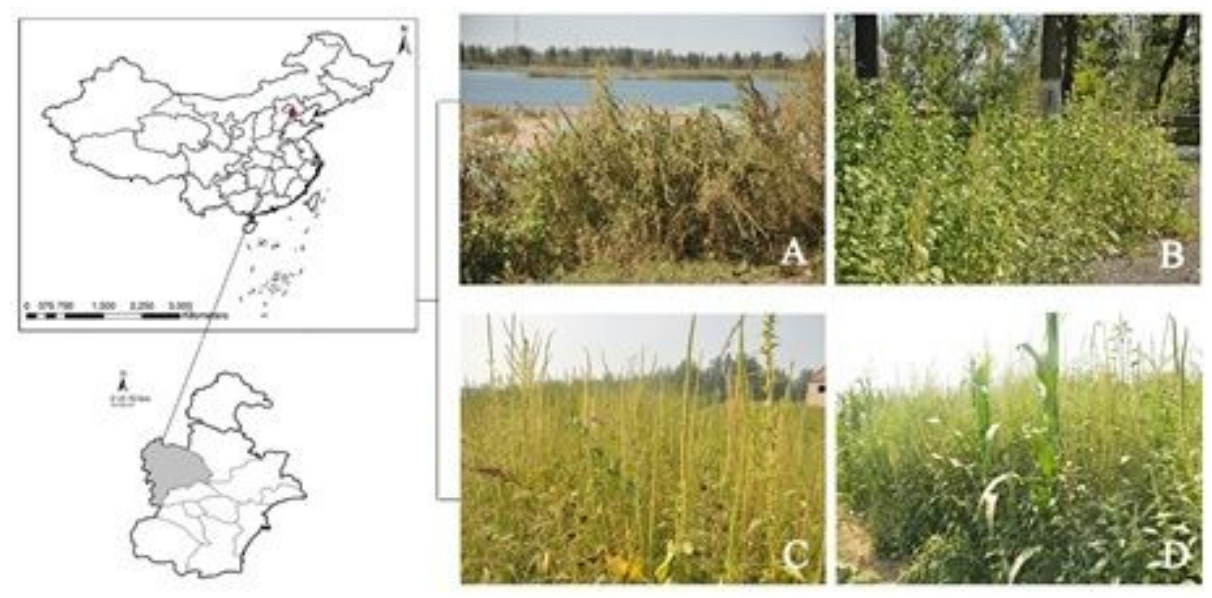

\section{Figure 1}

Location of research site and four types of sampling habitats (riverbank (A), roadside (B), wasteland (C) and farmland (D) habitats). The geographical map was created by Q-GIS Desktop 3.8.0 with GRASS 7.6.1 (www.qgis.org/en/site/), the habitat photos were taken by an Canon EOS 6D (Japan).
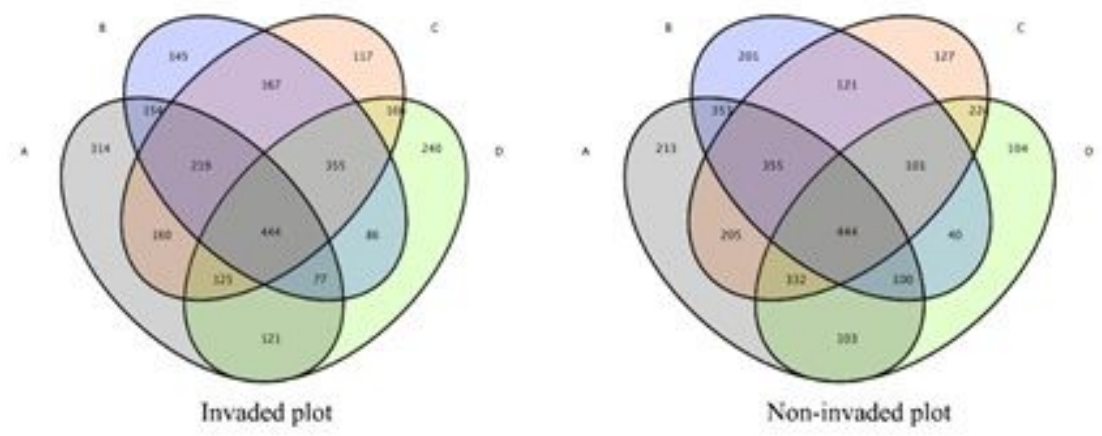

\section{Figure 2}

Venn diagrams of shared OTUs (number of OTUs) of invaded (left) and non-invaded (right) by A. palmeri plants under four sampling habitats. Letters in the figure, A: riverbank, B: roadside C: wasteland D: farmland. 


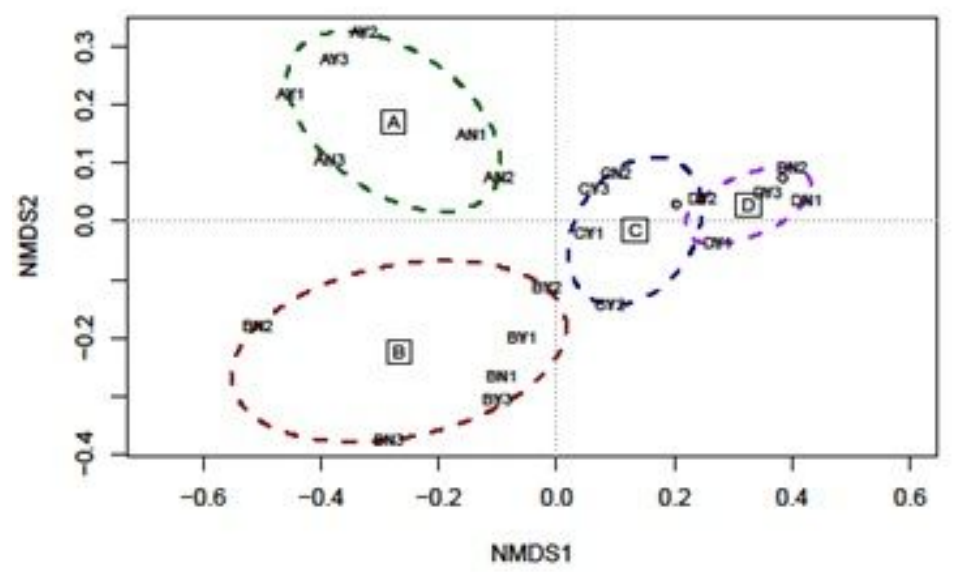

Figure 3

NMDS analysis of bacterial communities using Binary-Jaccard distances under four habitats.
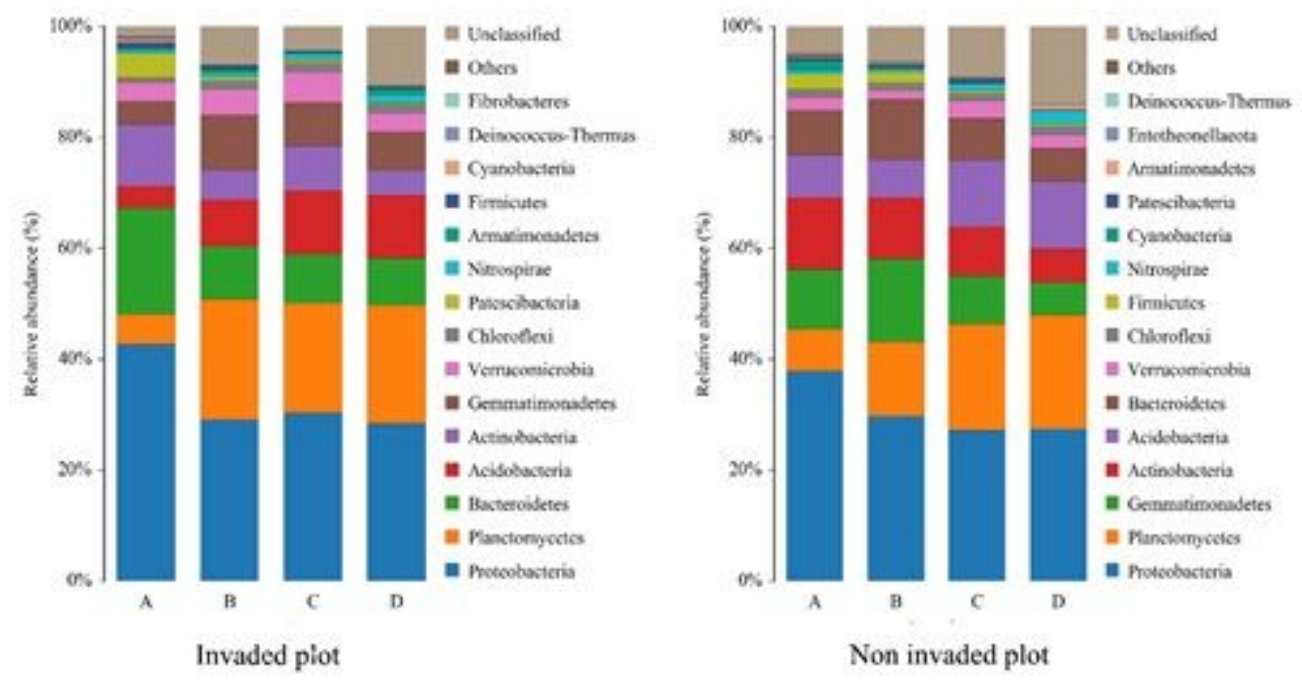

Figure 4

Phylum-level taxonomic composition of the bacterial community in the soil of A. palmeri invaded and non-invaded plots under four habitats. The legend at the top right shows the top 10 species classified by species abundance. The others are classified as Others, and the unannotated species are classified as Unclassified. 


\section{Cladogram}
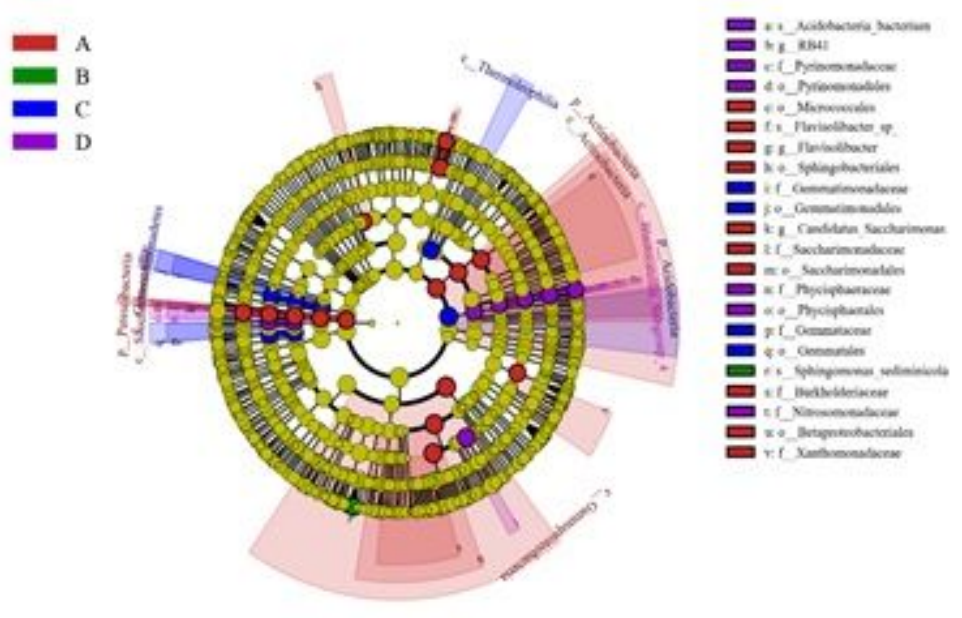

\section{Cladogram}

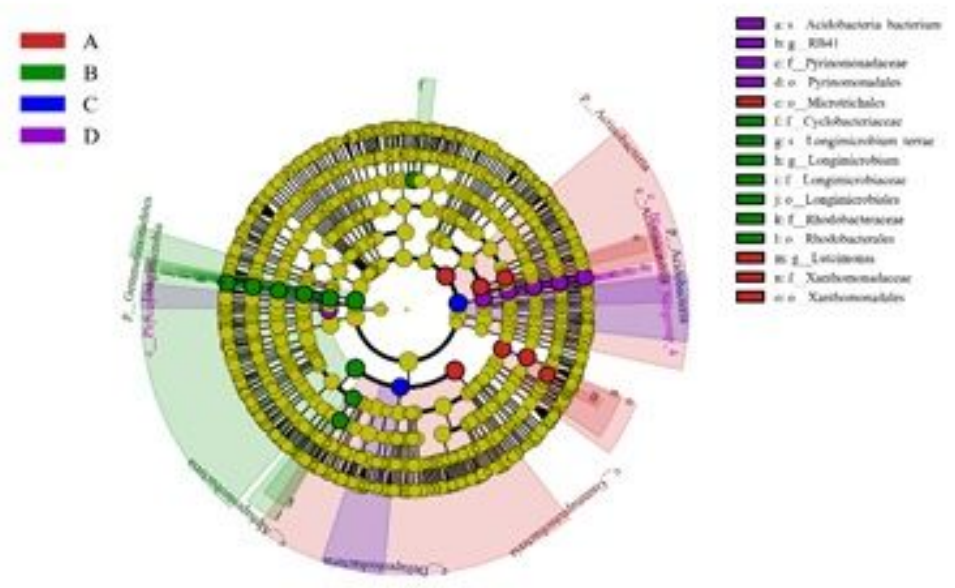

Figure 5

Cladograms via linear discriminate analysis (LDA) of effect size (LEfSe) with an LDA score higher than 4.0 of A. palmeri invasive (top) and non-invasive (bottom) treatments under heterogeneous habitats. Significant differences in microbial abundances according to taxa among habitats are represented by colored dots, Circles represent phylogenetic levels from kingdom to genus. 